\title{
Echinoderms of the Seaflower Biosphere Reserve: State of Knowledge and New Findings
}

\author{
Giomar H. Borrero-Pérez ${ }^{1 *}$, Milena Benavides-Serrato ${ }^{2}$, Néstor H. Campos ${ }^{2}$, \\ Elizabeth Galeano-Galeano ${ }^{3}$, Brigitte Gavio ${ }^{3,4}$, Jairo Medina ${ }^{3}$ and Alfredo Abril-Howard ${ }^{3}$ \\ ${ }^{1}$ Programa de Biodiversidad, Museo de Historia Natural Marina de Colombia, Instituto de Investigaciones Marinas y \\ Costeras Invemar, Santa Marta, Colombia, ${ }^{2}$ CECIMAR, Universidad Nacional de Colombia, Sede Caribe, Santa Marta, \\ Colombia, ${ }^{3}$ Universidad Nacional de Colombia, Sede Caribe, San Andrés, Colombia, ${ }^{4}$ Universidad Nacional de Colombia, \\ Sede Bogotá, Bogotá, Colombia
}

Keywords: taxonomic inventory, new records, distribution patterns, Crinoidea, Asteroidea, Ophiuroidea, Echinoidea, Holothuroidea

\section{BACKGROUND}

\section{OPEN ACCESS}

Edited by:

Sonia Bejarano,

Leibniz Centre for Tropical Marine

Research (LG), Germany

Reviewed by:

Renata Alitto,

Campinas State University, Brazil

Carolina Bastidas,

Massachusetts Institute of Technology, United States

*Correspondence: Giomar H. Borrero-Pérez

giomar.borrero@invemar.org.co; giomarborrero@gmail.com

Specialty section:

This article was submitted to Marine Evolutionary Biology, Biogeography and Species Diversity,

a section of the journal Frontiers in Marine Science

Received: 21 September 2018 Accepted: 26 March 2019 Published: 17 April 2019

Citation:

Borrero-Pérez GH,

Benavides-Serrato $M$, Campos NH, Galeano-Galeano E, Gavio B,

Medina J and Abril-Howard A (2019)

Echinoderms of the Seaflower

Biosphere Reserve: State of

Knowledge and New Findings.

Front. Mar. Sci. 6:188.

doi: 10.3389/fmars.2019.00188
Echinoderms are a charismatic marine invertebrate group that stand out for their beauty and color, but also for several attributes. Their morphological and biological characteristics represent innovations and particularities in animal evolution. Several species have proven to be key in the functioning of ecosystems and therefore in their conservation. Besides, some sea cucumbers and sea urchins are used for human consumption, being currently highly commercially important. In this paper we summarize the list of echinoderms in the SeaFlower Biosphere Reserve (SFBR) and their distribution patterns, including shallow and deep species. The SFBR was declared in November 2000 by UNESCO, and covers the extension of the department of Colombia "Archipiélago de San Andrés, Providencia y Santa Catalina," which has an approximate area of $180,000 \mathrm{~km}^{2}$ of which $<1 \%$ is emerged (CORALINA-INVEMAR, 2012). Within the SFBR is the Marine Protected Area Seaflower with $65,018 \mathrm{~km}^{2}$, declared in 2005 and divided in three sections: a northern section $\left(37,522 \mathrm{~km}^{2}\right)$ that includes Quitasueño, Serrana and Roncador as well as multiple deep submerged banks; a central section $\left(12,716 \mathrm{~km}^{2}\right)$ that includes Providencia and Santa Catalina; and a southern section $\left(14,780 \mathrm{~km}^{2}\right)$ encompassing San Andrés, Bolívar and Albuquerque among other banks (Figure 1). Although the sections share similar environments dominated by corals, transparent waters and oceanic features, each one has particular dynamics of fishing exploitation, which make them to require specific management actions (CORALINA-INVEMAR, 2012). To compile the data presented in this study we reviewed literature, specimen's records from museums, open access databases, and the species collected during recent samplings made in 2011 by Universidad Nacional de Colombia-Sede Caribe and the Seaflower Expeditions 2016 and 2017. This dataset provides a baseline for echinoderm species composition and geographic distribution, thus expanding the knowledge on biodiversity within the SFBR as part of the current SeaFlower Expeditions. It is essential to continue efforts to access these remote areas at SFBR, implement the use of new tools to evaluate their biodiversity (such as DNA barcoding) and define priority research topics for the conservation of marine biodiversity in this strategically located reserve.

\section{DATA DESCRIPTION}

\section{Data Collection}

We report here the dataset entitled "Echinoderms from the SeaFlower Biosphere Reserve" which updates the checklist presented by Borrero-Pérez et al. (2016) including new records from unpublished results of samplings conducted in 2011 by Universidad Nacional de Colombia-Sede Caribe, the Seaflower Expeditions 2016 and 2017, and open-access databases. 


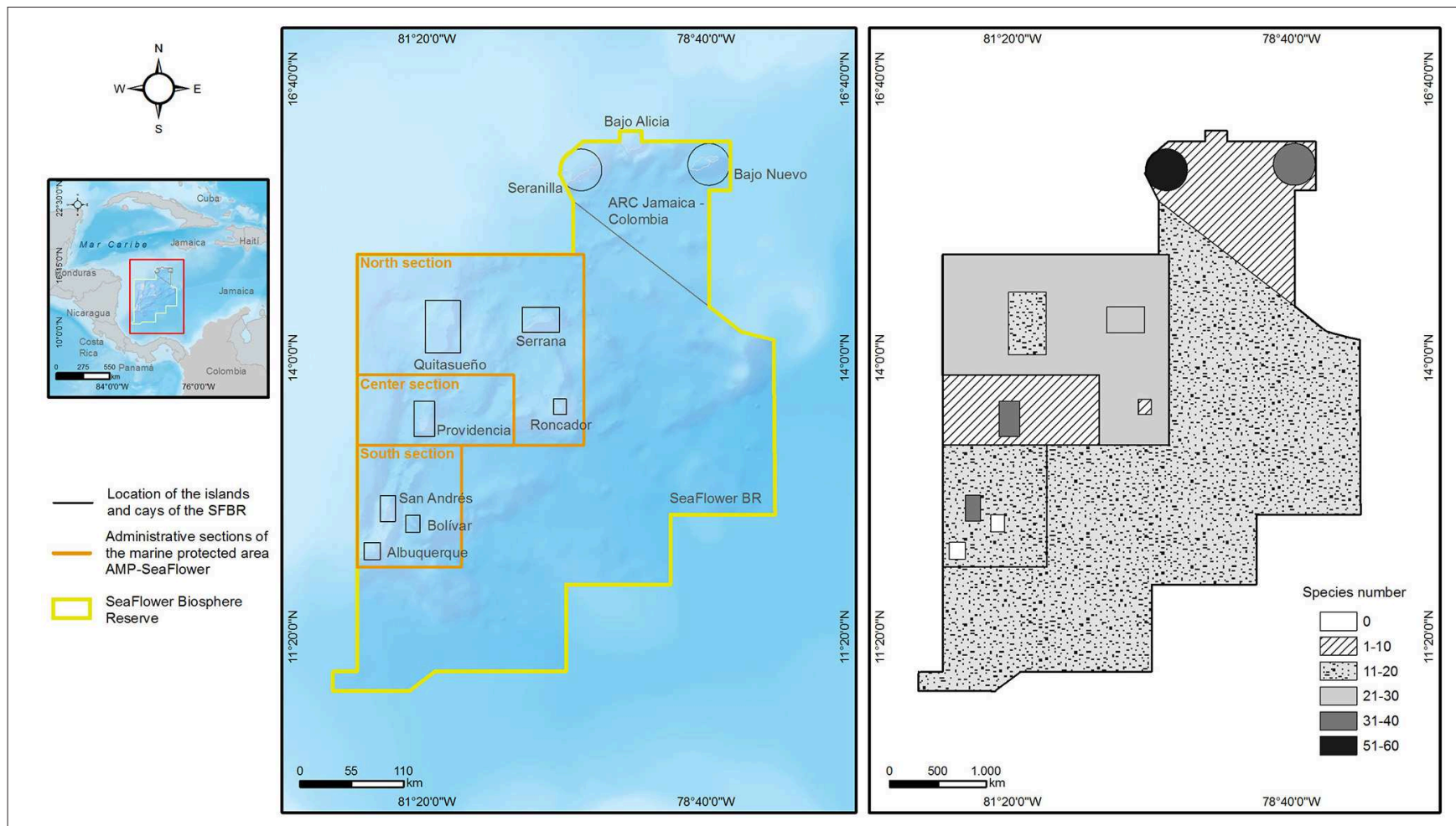

FIGURE 1 | Location of the SeaFlower Biosphere Reserve (SFBR), indicating the locations considered in the Checklist (left) and comparing the number of species registered in each one (right).

A descriptive analysis of species richness and spatial distribution patterns was made and some outcomes are presented.

This dataset compiles the lists of echinoderm species recorded within the SFBR and derived from: (i) Peer reviewed papers or published reports of several investigations, most of them supported by voucher specimens deposited at Museo de Historia Natural Marina de Colombia (MHNMC-INVEMAR), available through the Sistema de Información sobre Biodiversidad Marina (SIBM), and at the National Museum of Natural History (NMNH) (Clark, 1939; Quiñones, 1981; Clark and Downey, 1992; CORALINA, 2001; Diaz et al., 2003; García et al., 2003; Vélez, 2003; García-Hanse and Álvarez-León, 2007; Lasso, 2007a,b; Mejía-Ladino et al., 2008; Benavides-Serrato et al., 2011, 2013; Abril-Howard et al., 2012; Borrero-Pérez et al., 2012; INVEMAR-ANH, 2012; Vega-Sequeda et al., 2015; INVEMAR-SIBM $a^{1}, b^{2}, c^{3}$; $N_{M N H}^{4}$ ); (ii) The Ocean Biogeographic Information System (OBIS) data base; (iii) The

\footnotetext{
${ }^{1}$ INVEMAR -SIBM a. Acceso en línea en. Available online at: http://siam.invemar. org.co/sibm-busqueda-avanzada. Ecorregión SAN. Consultado en: 29-06-2015

${ }^{2}$ INVEMAR-SIBM b. Acceso en línea en. Available online at: http://siam.invemar. org.co/sibm-busqueda-avanzada. Proyecto Expedición Providencia I. Consultado en: 19-09-2018.

${ }^{3}$ INVEMAR-SIBM c. Acceso en línea en. Available online at: http://siam.invemar. org.co/sibm-busqueda-avanzada. Proyecto ANH ARC Jamaica. Consultado en: 17-07-2018

${ }^{4}$ NMNH. National Museum of Natural History. Acceso en línea en. Available online at: http://collections.nmnh.si.edu/search/iz/. Consultado en: 23-06-2015
}

Global Biodiversity of Information Facility (GBIF) data base; (iv) Biological records available at SIB Colombia reporting the unpublished results of samplings made in 2011 and the results of several projects developed during the Seaflower Expeditions 2016 and 2017 (UNAL (Universidad Nacional de Colombia), 2016; Benavides-Serrato et al., 2018a,b; Borrero-Pérez, 2018). The search at GBIF database was conducted using two polygons including the SFBR: Polygon $1(-80 \mathrm{~W} 11.8 \mathrm{~N},-78 \mathrm{~W} 11.8 \mathrm{~N}$, $-78 \mathrm{~W} 16.2 \mathrm{~N},-80 \mathrm{~W} 16.2 \mathrm{~N},-80 \mathrm{~W} 11.8 \mathrm{~N})$, Polygon $2(-82 \mathrm{~W}$ $11 \mathrm{~N},-80 \mathrm{~W} 11 \mathrm{~N},-80 \mathrm{~W} 15 \mathrm{~N},-82 \mathrm{~W} 15 \mathrm{~N},-82 \mathrm{~W} 11 \mathrm{~N})$. Using this search criteria we recovered new information formerly recorded as present in Nicaragua. The following nine fields are presented per species in this dataset:

taxonID and scientificNameID: These fields include a unique and stable-through-time alphanumeric identifier (taxonomic identifier) AphiaID provided by World Register of Marine Species (WoRMS).

locality: Specific localities were the species have been recorded in the SFBR are presented in this field. Fourteen localities were defined (Figure 1) considering the largest islands San Andrés (1), and Providencia and Santa Catalina (2); the Cay Islands Serranilla (3), Bajo Nuevo (4), Quitasueño (5), Serrana (6), Roncador (7), Albuquerque (8) and Bolivar (9); the Joint Regime Area Jamaica-Colombia (ARC Jamaica-Colombia, in Spanish) (10); the administrative sections of the SeaFlower marine protected area: North section (11), Central section (12) and South section (13) (CORALINA-INVEMAR, 2012), that were used to locate 
the species not recorded in the area near the islands or cays; and finally, the locality Seaflower BR (14), where all the recorded species outside of the previously mentioned localities and sections were located.

locationID and coordinates: Fields based on the Marine regions Gazetteer (http://www.marineregions.org/ gazetteer.php).

catalogNumber and collectionCode: These fields list cataloged lots available for each species, indicating the museums were they are deposited.

basisOfRecord: This field indicates PreservedSpecimens and HumanObservations, which includes records from literature.

bibliographicCitation: This field includes the reference that explicitly mentions the species in the RBSF, usually with voucher specimens.

References: This field includes references that have compiled echinoderm species records in the SFBR or in the Colombian Caribbean Sea.

institutionCode: This field includes the name (or acronym) in use by the institution having custody of the object(s) or information referred to in the record.

eventDate: This field includes the date of collection of the specimens recorded. Wherever the exact collection date is unknown, a comment appears in the field eventRemarks.

\section{Echinoderms Species and Distribution Patterns}

A total of 148 echinoderm species were found within the SFBR. Ophiuroidea was the most species-rich class (44 species), followed by Asteroidea (38), Echinoidea (31), Holothuroidea (26) and finally Crinoidea (9). Considering the revision by Borrero-Pérez et al. (2016) as a baseline, there are 23 new records for the SFBR; eight of them are shallow water species based on unpublished results of samplings made in 2011 by UNAL and SeaFlower Expeditions 2016, 2017 at Quitasueño, Roncador, Serrana and Serranilla (Benavides-Serrato et al., 2018a,b; Borrero-Pérez, 2018); and 15 are deep water species located mostly in SeaFlower BR (13 species) and in the North section (2) localities, recorded from GBIF data base. As these deep species were assigned to Nicaragua, they were not considered in previous publications. The majority of these deep water specimens were collected by research vessels Alaminos and Gyre during the 1960's and 1970's (Prestridge, 2016). On a smaller scale, the importance of the expeditions carried out recently is reflected, considering that the increase in the number of species for Quitasueño and Serrana was $80 \%$, Serranilla 25\%, and Roncador was 100\% (Table 1).

The echinoderms recorded for the first time in the SFBR are the shallow water species: Astropecten duplicatus, Copidaster lymani, Ophioderma cinerea, Ophiolepis impressa, Ophiolepis paucispina, Arbacia punctulata, Mellita quinquiesperforata, Brissus unicolor; and the deep water species: Plinthaster dentatus, Zoroaster fulgens, Hymenaster modestus, Litonotaster intermedius, Dytaster insignis, Benthopecten simplex,
Calyptraster personatus, Ophiothrix pallida, Deima validum validum, Mesothuria verrilli, Mesothuria maroccana, Molpadia cubana, Psychropotes depressa, Benthodytes typica, and Benthodytes lingua (Table 1). Ten of these species (in bold), are also first records for the Colombian Caribbean Sea.

The highest number of species were recorded in shallow waters near San Andrés (36 species), and Providencia and Santa Catalina islands (37), which is expected given their accessibility and the greatest sampling effort in those islands. However, Serranilla which is the farthest locality from Colombian continent cost, is currently the richest with 54 species. This locality has been sampled several times and this number of species includes shallow and deep environments. Some of the Serranilla records came from research cruises previous to 1970's; from expeditions carried out by INVEMAR in 2011 to characterize shallow and deep ecosystems (INVEMARANH, 2012; Vega-Sequeda et al., 2015); and from the recent Seaflower Expedition in 2017 (Benavides-Serrato et al., 2018b; Borrero-Pérez, 2018). Albuquerque and Bolivar, located nearest to San Andres, showed no echinoderm records (Figure 1; Table 1).

Although deep environments have been relatively less studied in the SFBR, except by the research project focused on ARC Jamaica-Colombia (INVEMAR-ANH, 2012), 48\% of the species currently recorded in the SFBR are distributed only or mainly below $200 \mathrm{~m}$ in depth. These species records are the result of some sampling stations of research cruises like the R/V Albatross (1884), Presidential Cruise (1938), R/V Oregon (1957, 1964), R/V Pillsbury (1971), R/V Alaminos, and R/V Gyre during the 1960's and 1970's. The samples of these cruises are deposited mostly in the Smithsonian NMNH and most of them were included in the catalogs of the echinoderms of Colombia and other publications (Clark, 1939; Clark and Downey, 1992; Benavides-Serrato et al., 2011; Borrero-Pérez et al., 2012; Prestridge, 2016). Given that samples from R/V Gyre during the 1960's and 1970's were assigned to Nicaragua they had not been considered in previous publications (Orrell and Hollowell, 2018).

Most of the deep water species (75\%) have a single record in the reserve, which is expected considering that samplings at deep water are restricted to some of the deep banks in the SFBR, such as those sampled around Serranilla and Bajo Nuevo by INVEMAR-ANH (2012); or restricted to some stations during the research cruises previously mentioned. In the case of shallow water species, $42 \%$ (32 species) have a single record (one locality), 42\% (32 species) 2 to 4 , and 16\% (13 species) 5 to 10 records. Among them only 17 species (22\%) are distributed throughout the reserve, recorded in several localities or at least in Serranilla and/or Bajo Nuevo (the northernmost localities) and in one locality of the South section. These species are the sea star Oreaster reticulatus (reported in 4 localities), the brittle stars Ophiocoma echinata (7), Ophiothrix oerstedii (6), Ophioderma appressa (6) and O. rubicunda (4); the sea urchins Diadema antillarum (8), Eucidaris tribuloides (7), Echinometra lucunter lucunter (7), E. viridis (6), Tripneustes 
TABLE 1 | Species of echinoderms of the SeaFlower Biosphere Reserve.

\begin{tabular}{|c|c|c|c|c|c|c|c|c|c|c|c|c|c|c|}
\hline Species & $\begin{array}{l}\text { South } \\
\text { section }\end{array}$ & San Andrés & Bolivar & Albuquerque & $\begin{array}{l}\text { Central } \\
\text { section }\end{array}$ & $\begin{array}{l}\text { Providencia } \\
\text { and Santa } \\
\text { Catalina }\end{array}$ & $\begin{array}{l}\text { North } \\
\text { section }\end{array}$ & Quitasueño & Serrana & Roncador & $\begin{array}{l}\text { Bajo } \\
\text { Nuevo }\end{array}$ & Serranilla & $\begin{array}{c}\text { ARC } \\
\text { Jamaica- } \\
\text { Colombia }\end{array}$ & $\begin{array}{c}\text { SeaFlower } \\
\text { Biosphere } \\
\text { Reserve }\end{array}$ \\
\hline Caryometra atlantidis & & & & & & & & & & & $x$ & & & \\
\hline Trichometra cubensis & & & & & & & $x$ & & & & $x$ & & & \\
\hline Davidaster discoideus & & $x$ & & & & & & & & & & & & \\
\hline Davidaster rubiginosus & & $x$ & & & & $x$ & & & & & & & & \\
\hline Crinometra brevipinna & & & & & & & & & & & $x$ & & & \\
\hline Stylometra spinifera & & & & & & & & & & & $x$ & & & \\
\hline $\begin{array}{l}\text { Endoxocrinus } \\
\text { (Endoxocrinus) parrae } \\
\text { parrae }\end{array}$ & & & & & & & & & & & $x$ & & & \\
\hline Democrinus conifer & & & & & & & $x$ & & & & & & & \\
\hline Democrinus rawsonii & & & & & & & $x$ & & & & & & & \\
\hline $\begin{array}{l}\text { Luidia alternata } \\
\text { alternata }\end{array}$ & & & & & & & & & & & & $x$ & & \\
\hline Luidia barbadensis & & & & & & & $x$ & & & & & & & \\
\hline Luidia clathrata & & & & & & & & & & & & $x$ & & \\
\hline Luidia senegalensis & & & & & & & & & & & & $x$ & & \\
\hline $\begin{array}{l}\text { Astropecten } \\
\text { americanus }\end{array}$ & & & & & & & $x$ & & & & & & & \\
\hline Astropecten antillensis & & & & & & & & & & & & $x$ & & \\
\hline Astropecten articulatus & & & & & & & & & & & & $x$ & & \\
\hline Astropecten cingulatus & & & & & & & $x$ & & & & & & & \\
\hline $\begin{array}{l}\text { Astropecten } \\
\text { duplicatus* }\end{array}$ & & & & & & & & $\mathbf{R}$ & & & & & & \\
\hline Astropecten nitidus & & & & & & & $x$ & & & & & & & \\
\hline Dytaster insignis* & & & & & & & & & & & & & & $\mathbf{R}$ \\
\hline $\begin{array}{l}\text { Persephonaster } \\
\text { echinulatus }\end{array}$ & & & & & $x$ & & & & & & & & & \\
\hline $\begin{array}{l}\text { Pseudarchaster gracilis } \\
\text { gracilis }\end{array}$ & & & & & $x$ & & & & & & & & & \\
\hline $\begin{array}{l}\text { Benthopecten } \\
\text { simplex* }\end{array}$ & & & & & & & & & & & & & & $\mathbf{R}$ \\
\hline $\begin{array}{l}\text { Cheiraster } \\
\text { (Barbadosaster) } \\
\text { echinulatus }\end{array}$ & & & & & & & $x$ & & & & & & & \\
\hline $\begin{array}{l}\text { Cheiraster } \\
\text { (Christopheraster) } \\
\text { mirabilis }\end{array}$ & & & & & $x$ & & & & & & & & & \\
\hline Asterinides folium & $x$ & & & & & & & & & & & & & \\
\hline
\end{tabular}


TABLE 1 | Continued

\begin{tabular}{|c|c|c|c|c|c|c|c|c|c|c|c|c|c|c|}
\hline Species & $\begin{array}{l}\text { South } \\
\text { section }\end{array}$ & San Andrés & Bolivar & Albuquerque & $\begin{array}{l}\text { Central } \\
\text { section }\end{array}$ & $\begin{array}{l}\text { Providencia } \\
\text { and Santa } \\
\text { Catalina }\end{array}$ & $\begin{array}{l}\text { North } \\
\text { section }\end{array}$ & Quitasueño & Serrana & Roncador & $\begin{array}{l}\text { Bajo } \\
\text { Nuevo }\end{array}$ & Serranilla & $\begin{array}{c}\text { ARC } \\
\text { Jamaica- } \\
\text { Colombia }\end{array}$ & $\begin{array}{c}\text { SeaFlower } \\
\text { Biosphere } \\
\text { Reserve }\end{array}$ \\
\hline Stegnaster wesseli & $x$ & & & & & & & & & & & & & \\
\hline Marginaster pectinatus & & & & & & & & & & & $x$ & & & \\
\hline Anthenoides peircei & & & & & & & $x$ & & & & $x$ & & & \\
\hline $\begin{array}{l}\text { Apollonaster } \\
\text { yucatanensis }\end{array}$ & & & & & & & & & & & & & & $x$ \\
\hline Circeaster americanus & & & & & $x$ & & $x$ & & & & & & & \\
\hline Nymphaster arenatus & & & & & $x$ & & $x$ & & & & & & & \\
\hline Rosaster alexandri & & & & & & & $x$ & & & & & & & \\
\hline Linckia bouvieri & & & & & & & & & $x$ & & & & & \\
\hline Linckia guildingi & & & & & & $x$ & & & & & & $\mathbf{R}$ & & \\
\hline Ophidiaster guildingi & & & & & & $x$ & & & $\mathbf{R}$ & & & & & \\
\hline Tamaria halperni & & & & & & & & $x$ & $x$ & & & $x$ & & \\
\hline Oreaster reticulatus & & $x$ & & & & $x$ & & & $\mathbf{R}$ & & & $x$ & & \\
\hline Copidaster lymani * & & & & & & & & & & & & $\mathbf{R}$ & & \\
\hline Plinthaster dentatus ${ }^{\star}$ & & & & & & & $\mathbf{R}$ & & & & & & & \\
\hline $\begin{array}{l}\text { Litonotaster } \\
\text { intermedius }^{\star}\end{array}$ & & & & & & & & & & & & & & $\mathbf{R}$ \\
\hline $\begin{array}{l}\text { Calyptraster } \\
\text { personatus* }\end{array}$ & & & & & & & & & & & & & & $\mathbf{R}$ \\
\hline $\begin{array}{l}\text { Hymenaster } \\
\text { modestus }\end{array}$ & & & & & & & & & & & & & & $\mathbf{R}$ \\
\hline $\begin{array}{l}\text { Pteraster militaroides } \\
\text { militaroides }\end{array}$ & & & & & $x$ & & & & & & & & & \\
\hline $\begin{array}{l}\text { Echinaster (Othilia) } \\
\text { brasiliensis }\end{array}$ & & & & & & & & & $x$ & & & & & \\
\hline Henricia antillarum & & & & & & & $x$ & & & & & & $x$ & \\
\hline Zoroaster fulgens* & & & & & & & & & & & & & & $\mathbf{R}$ \\
\hline Astrophyton muricatum & & & & & & $x$ & & & & & & $x$ & & \\
\hline Ophiomyxa flaccida & & & & & & $x$ & & & & & & & & \\
\hline Ophiacantha bidentata & & & & & & & & & & & $x$ & & $x$ & \\
\hline Ophiocamax hystrix & & & & & & & & & & & $x$ & & & \\
\hline Ophiomitra valida & & & & & & & & & & & $x$ & & $x$ & \\
\hline Ophiopristis hirsuta & & & & & & & & & & & $x$ & $x$ & $x$ & \\
\hline Ophiotreta cf. sertata & & & & & & & & & & & $x$ & & $x$ & \\
\hline Ophiocoma echinata & $x$ & $x$ & & & & $x$ & & $\mathbf{R}$ & $\mathbf{R}$ & $\mathbf{R}$ & & $\mathbf{R}$ & & \\
\hline Ophiocoma pumila & & & & & & & & $\mathbf{R}$ & $\mathbf{R}$ & & & $x$ & & \\
\hline Ophiocoma wendtii & & & & & & $x$ & & $\mathbf{R}$ & $\mathbf{R}$ & & & $\mathbf{R}$ & & \\
\hline
\end{tabular}


TABLE 1 | Continued

\begin{tabular}{|c|c|c|c|c|c|c|c|c|c|c|c|c|c|c|}
\hline Species & $\begin{array}{l}\text { South } \\
\text { section }\end{array}$ & San Andrés & Bolivar & Albuquerque & $\begin{array}{l}\text { Central } \\
\text { section }\end{array}$ & $\begin{array}{l}\text { Providencia } \\
\text { and Santa } \\
\text { Catalina }\end{array}$ & $\begin{array}{l}\text { North } \\
\text { section }\end{array}$ & Quitasueño & Serrana & Roncador & $\begin{array}{l}\text { Bajo } \\
\text { Nuevo }\end{array}$ & Serranilla & $\begin{array}{c}\text { ARC } \\
\text { Jamaica- } \\
\text { Colombia }\end{array}$ & $\begin{array}{c}\text { SeaFlower } \\
\text { Biosphere } \\
\text { Reserve }\end{array}$ \\
\hline Bathypectinura heros & & & & & & & & & & & $x$ & & & \\
\hline Ophioderma appressa & $x$ & $x$ & & & & $x$ & & $\mathbf{R}$ & $\mathbf{R}$ & & & $\mathbf{R}$ & & \\
\hline $\begin{array}{l}\text { Ophioderma } \\
\text { brevicauda }\end{array}$ & & $x$ & & & & & & & & & & & & \\
\hline Ophioderma brevispina & & & & & & & & & & & & $x$ & & \\
\hline Ophioderma cinerea* & & & & & & & & & $\mathbf{R}$ & & & $\mathbf{R}$ & & \\
\hline Ophioderma rubicunda & & $x$ & & & & & & $\mathbf{R}$ & $\mathbf{R}$ & & & $\mathbf{R}$ & & \\
\hline Ophiopaepale goesiana & & & & & & & & & & & $x$ & & & \\
\hline $\begin{array}{l}\text { Ophiomyces } \\
\text { frutectosus }\end{array}$ & & & & & & & & & & & $x$ & & & \\
\hline Amphiodia pulchella & & $x$ & & & & $x$ & & & & & & & & \\
\hline Amphiodia trychna & & $x$ & & & & & & & & & & & & \\
\hline Amphipholis gracillima & & $x$ & & & & & & & & & & & & \\
\hline Amphipholis januarii & & $x$ & & & & $\mathrm{x}$ & & & & & & & & \\
\hline Amphipholis squamata & & $x$ & & & & $x$ & & & & & & & & \\
\hline Amphiura stimpsonii & & $x$ & & & & & & & & & & & & \\
\hline Microphiopholis atra & & & & & & & & & & & & $x$ & & \\
\hline Ophionephthys limicola & & $x$ & & & & & & & & & & & & \\
\hline $\begin{array}{l}\text { Ophiophragmus } \\
\text { pulcher }\end{array}$ & & $x$ & & & & $x$ & & & & & & & & \\
\hline $\begin{array}{l}\text { Ophiostigma } \\
\text { isocanthum }\end{array}$ & & $x$ & & & & $x$ & & & & & & & & \\
\hline Ophiostigma siva & & $x$ & & & & $x$ & & & & & & & & \\
\hline $\begin{array}{l}\text { Ophiothrix (Ophiothrix) } \\
\text { angulata }\end{array}$ & & $x$ & & & & $x$ & & & & & & & & \\
\hline Ophiothrix lineata & & & & & & & & & & & & $x$ & & \\
\hline $\begin{array}{l}\text { Ophiothrix (Ophiothrix) } \\
\text { oerstedii }\end{array}$ & $x$ & $x$ & & & & $x$ & & & $\mathbf{R}$ & & $x$ & $x$ & & \\
\hline Ophiothrix pallida* & & & & & & & $\mathbf{R}$ & & & & & & & \\
\hline $\begin{array}{l}\text { Ophiothrix } \\
\text { (Acanthophiothrix) } \\
\text { suensoni }\end{array}$ & & & & & & $x$ & & & & $\mathbf{R}$ & $x$ & $x$ & & \\
\hline Ophiactis savignyi & & $x$ & & & & & & & & & & & & \\
\hline Ophionereis reticulata & & & & & & $x$ & & & $\mathbf{R}$ & & $x$ & $\mathbf{R}$ & & \\
\hline Ophiolepis elegans & & $x$ & & & & & & & & & & & & \\
\hline Ophiolepis impressa * & & & & & & & & $\mathbf{R}$ & $\mathbf{R}$ & $\mathbf{R}$ & & $\mathbf{R}$ & & \\
\hline Ophiolepis paucispina* & & & & & & & & & & & & $\mathbf{R}$ & & \\
\hline
\end{tabular}


TABLE 1 | Continued

\begin{tabular}{|c|c|c|c|c|c|c|c|c|c|c|c|c|c|c|}
\hline Species & $\begin{array}{l}\text { South } \\
\text { section }\end{array}$ & San Andrés & Bolivar & Albuquerque & $\begin{array}{l}\text { Central } \\
\text { section }\end{array}$ & $\begin{array}{c}\text { Providencia } \\
\text { and Santa } \\
\text { Catalina }\end{array}$ & $\begin{array}{l}\text { North } \\
\text { section }\end{array}$ & Quitasueño & Serrana & Roncador & $\begin{array}{l}\text { Bajo } \\
\text { Nuevo }\end{array}$ & Serranilla & $\begin{array}{c}\text { ARC } \\
\text { Jamaica- } \\
\text { Colombia }\end{array}$ & $\begin{array}{c}\text { SeaFlower } \\
\text { Biosphere } \\
\text { Reserve }\end{array}$ \\
\hline Ophiothyreus goesi & & & & & & & & $x$ & & & & & & \\
\hline $\begin{array}{l}\text { Ophiomusium } \\
\text { acuferum }\end{array}$ & & & & & & & & & & & $x$ & & $x$ & \\
\hline $\begin{array}{l}\text { Ophiomusium } \\
\text { eburneum }\end{array}$ & & & & & & & & & & & $x$ & & & \\
\hline Ophiomusium testudo & & & & & & & & & & & $x$ & $x$ & & \\
\hline Ophiomusium validum & & & & & & & & & & & $x$ & & & \\
\hline Eucidaris tribuloides & & $x$ & & & & $x$ & & $x$ & $\mathbf{R}$ & & $x$ & $x$ & $x$ & \\
\hline Stylocidaris affinis & & & & & & & $x$ & & & & & & & \\
\hline Stylocidaris lineata & & & & & & & & & & & $\mathrm{x}$ & $x$ & & \\
\hline Araeosoma belli & & & & & & & $x$ & & & & & & & \\
\hline Aspidodiadema jacobyi & & & & & & & $x$ & & & & & & & \\
\hline Diadema antillarum & $x$ & $x$ & & & & $x$ & & $\mathbf{R}$ & $\mathbf{R}$ & & $x$ & $x$ & $x$ & \\
\hline Bathysalenia goesiana & & & & & & & & & & & $x$ & $x$ & & \\
\hline Arbacia punctulata* & & & & & & & & $\mathbf{R}$ & & & & & & \\
\hline Coelopleurus floridanus & & & & & & & & & $x$ & & & & & \\
\hline Trigonocidaris albida & & & & & & & & & & & $x$ & & $x$ & \\
\hline $\begin{array}{l}\text { Lytechinus variegatus } \\
\text { variegatus }\end{array}$ & & $x$ & & & & $x$ & & & & & & $x$ & & \\
\hline Lytechinus williamsi & & $x$ & & & & & & & & & & & & \\
\hline Tripneustes ventricosus & $x$ & $x$ & & & & $x$ & & $\mathbf{R}$ & $\mathbf{R}$ & & & $x$ & & \\
\hline $\begin{array}{l}\text { Echinometra lucunter } \\
\text { lucunter }\end{array}$ & $x$ & $x$ & & & & $x$ & & $\mathbf{R}$ & $\mathbf{R}$ & & $x$ & $x$ & & \\
\hline Echinometra viridis & & $x$ & & & & $x$ & & $\mathbf{R}$ & $\mathbf{R}$ & & $\mathrm{x}$ & $x$ & & \\
\hline $\begin{array}{l}\text { Echinoneus } \\
\text { cyclostomus }\end{array}$ & & $x$ & & & & & & & & & & & & \\
\hline Clypeaster lamprus & & & & & & & $x$ & $x$ & & & & & & \\
\hline Clypeaster rosaceus & & $x$ & & & & $x$ & & & & & & $x$ & & \\
\hline $\begin{array}{l}\text { Clypeaster } \\
\text { subdepressus }\end{array}$ & & $x$ & & & & & & & & & & & & \\
\hline $\begin{array}{l}\text { Echinocyamus } \\
\text { grandiporus }\end{array}$ & & & & & & $x$ & & & & & $x$ & $x$ & & \\
\hline Encope emarginata & & & & & & & & & & & & $x$ & & \\
\hline Leodia sexiesperforata & $x$ & & & & & $x$ & & & & & & $\mathbf{R}$ & & \\
\hline $\begin{array}{l}\text { Mellita } \\
\text { quinquiesperforata* }\end{array}$ & & & & & & & & & $\mathbf{R}$ & & & & & \\
\hline Conolampas sigsbei & & & & & & & $x$ & & & & $x$ & & & \\
\hline
\end{tabular}


TABLE 1 | Continued

\begin{tabular}{|c|c|c|c|c|c|c|c|c|c|c|c|c|c|c|}
\hline Species & $\begin{array}{l}\text { South } \\
\text { section }\end{array}$ & San Andrés & Bolivar & Albuquerque & $\begin{array}{l}\text { Central } \\
\text { section }\end{array}$ & $\begin{array}{c}\text { Providencia } \\
\text { and Santa } \\
\text { Catalina }\end{array}$ & $\begin{array}{l}\text { North } \\
\text { section }\end{array}$ & Quitasueño & Serrana & Roncador & $\begin{array}{l}\text { Bajo } \\
\text { Nuevo }\end{array}$ & Serranilla & $\begin{array}{c}\text { ARC } \\
\text { Jamaica- } \\
\text { Colombia }\end{array}$ & $\begin{array}{c}\text { SeaFlower } \\
\text { Biosphere } \\
\text { Reserve }\end{array}$ \\
\hline Brissus unicolor* & & & & & & & & & & & & $\mathbf{R}$ & & \\
\hline $\begin{array}{l}\text { Meoma ventricosa } \\
\text { ventricosa }\end{array}$ & & & & & & $x$ & & & $\mathbf{R}$ & & & $x$ & & \\
\hline Plagiobrissus grandis & & $x$ & & & & & & & & & & & & \\
\hline Palaeobrissus hilgardi & & & & & & & & & & & $x$ & $x$ & & \\
\hline $\begin{array}{l}\text { Linopneustes } \\
\text { longispinus }\end{array}$ & & & & & & & $\mathrm{x}$ & & & & & & & \\
\hline Agassizia excentrica & & & & & & & & & & & $x$ & & & \\
\hline Heterobrissus hystrix & & & & & & & $x$ & & & & & & & \\
\hline Ocnus suspectus & & & & & & & & & & & & $x$ & & \\
\hline $\begin{array}{l}\text { Thyonella } \\
\text { sabanillaensis }\end{array}$ & & & & & & & & & & & & $x$ & & \\
\hline Pentamera pulcherrima & & & & & & & & & & & & $x$ & & \\
\hline Lissothuria braziliensis & & & & & & & & & & & & $x$ & & \\
\hline Astichopus multifidus & & & & & & & & & $\mathbf{R}$ & & & $x$ & & \\
\hline $\begin{array}{l}\text { Isostichopus } \\
\text { badionotus }\end{array}$ & & $x$ & & & & $x$ & & $\mathbf{R}$ & $\mathbf{R}$ & & & $x$ & $x$ & \\
\hline $\begin{array}{l}\text { Deima validum } \\
\text { validum* }\end{array}$ & & & & & & & & & & & & & & $\mathbf{R}$ \\
\hline Actinopyga agassizii & & & & & & $x$ & & & & & & $\mathbf{R}$ & & \\
\hline $\begin{array}{l}\text { Holothuria (Cystipus) } \\
\text { cubana }\end{array}$ & & & & & & $x$ & & & & & & & & \\
\hline $\begin{array}{l}\text { Holothuria (Halodeima) } \\
\text { floridana }\end{array}$ & & $x$ & & & & $x$ & & & & & & & & \\
\hline $\begin{array}{l}\text { Holothuria (Halodeima) } \\
\text { grisea }\end{array}$ & & & & & & & & & & & & $x$ & & \\
\hline $\begin{array}{l}\text { Holothuria (Halodeima) } \\
\text { mexicana }\end{array}$ & & $x$ & & & & $x$ & & $\mathbf{R}$ & $\mathbf{R}$ & $\mathbf{R}$ & $x$ & $x$ & & \\
\hline $\begin{array}{l}\text { Holothuria } \\
\text { (Platyperona) parvula }\end{array}$ & $x$ & & & & & & & & & & & $\mathbf{R}$ & & \\
\hline $\begin{array}{l}\text { Holothuria } \\
\text { (Selenkothuria) } \\
\text { glaberrima }\end{array}$ & $x$ & & & & & & & & & & & $x$ & & \\
\hline $\begin{array}{l}\text { Holothuria } \\
\text { (Thymiosycia) arenicola }\end{array}$ & & & & & & & & & & & & $x$ & & \\
\hline $\begin{array}{l}\text { Holothuria } \\
\text { (Thymiosycia) } \\
\text { impatiens }\end{array}$ & & & & & & $x$ & & & & & & & & \\
\hline
\end{tabular}


TABLE 1 | Continued

\begin{tabular}{|c|c|c|c|c|c|c|c|c|c|c|c|c|c|c|}
\hline Species & $\begin{array}{l}\text { South } \\
\text { section }\end{array}$ & San Andrés & Bolivar & Albuquerque & $\begin{array}{l}\text { Central } \\
\text { section }\end{array}$ & $\begin{array}{c}\text { Providencia } \\
\text { and Santa } \\
\text { Catalina }\end{array}$ & $\begin{array}{l}\text { North } \\
\text { section }\end{array}$ & Quitasueño & Serrana & Roncador & $\begin{array}{c}\text { Bajo } \\
\text { Nuevo }\end{array}$ & Serranilla & $\begin{array}{c}\text { ARC } \\
\text { Jamaica- } \\
\text { Colombia }\end{array}$ & $\begin{array}{c}\text { SeaFlower } \\
\text { Biosphere } \\
\text { Reserve }\end{array}$ \\
\hline
\end{tabular}

Holothuria

x

$\mathbf{R}$

(Thymiosycia) thomasi

Holothuria

(Vaneyothuria)

lentiginosa

Mesothuria verrilli`

Mesothuria

maroccana*

Euapta lappa

Synaptula hydriformis

Molpadia cubana

Psychropotes

depressa*

\section{Benthodytes typica*}

Benthodytes lingua

Total species by

Borrero-Pérez et al.

(2016)

Total first records per

locality

Total

0

0

6

$37 \quad 22$

$2 \quad 14$

$22 \quad 4$

4

X indicates presence of the species accounted by Borrero-Pérez et al. (2016); R indicates first records for a locality; asterisk (") in the species column indicates new records for SeaFlower Biosphere Reserve; and bold letter denote new records for Colombian Caribbean Sea. 
ventricosus (6), Clypeaster rosaceus (3), Leodia sexiesperforata (3) and Lytechinus variegatus variegatus (3); and the sea cucumbers Holothuria mexicana (7), Isostichopus badionotus (6), H. parvula (2), and H. glaberrima (2). Most of these species are also the most frequent, being recorded in more than 4 localities, and some of them occur in large densities. However, little is known about the local population densities and distribution of some species (e.g., Diadema antillarum) that have proven to be key in the functioning of reef ecosystems (Lessios, 2016) and I. badionotus and H. mexicana currently considered the most commercially important sea cucumbers in the Caribbean Sea (Guzman and Guevara, 2002; Toral-Granda, 2008). The high percentage of species records only in one locality likely are related with sampling effort. For example the widely-distributed and common family Amphiuridae is almost restricted to San Andres and Providencia and Santa Catalina Islands localities. Most amphiurids live buried in soft bottoms or hidden in different structures, extending their arms toward the surface to feed (Hendler et al., 1995). The records of this family in the SFBR come from a single study focused on this group using a specific sampling technique. More samplings are needed to complete the inventory and to better understand the spatial pattern of echinoderm diversity, including localities where the species composition is practically unknown, such as Albuquerque and Bolivar cays, and focusing on different habitats.

Knowledge on the geographic distribution of Echinoderm species throughout the SFBR is a prerequisite to understand the spatial patterns of larval connectivity and the role of SFBR in the Caribbean-wide Echinoderm population dynamics. Based on this knowledge it will be possible to define priority research topics for the conservation of marine biodiversity in this strategically located reserve for the Caribbean Sea. Currently, only the $53 \%$ of the species previously registered in the Colombian Caribbean (Benavides-Serrato et al., 2013), and 27\% of the species from the Caribbean Sea and the Gulf of Mexico (Alvarado-Barrientos and Solís-Marín, 2013) are recorded in the SFBR.

Finally, most of the species (88\%) included in this study have vouchers deposited in several biological collections, mostly in the Museo de Historia Natural Marina de Colombia (MHNMC-INVEMAR), the National Museum of Natural History-Smithsonian (NMNH-Smithsonian), and others, making a more reliable inventory. Physical evidence of each species is fundamental in taxonomy because it allows to unify criteria for the identification, especially of species or groups with variable characteristics, and dubious identifications.

\section{Re-Use Potential}

The checklist presented here provides an updated inventory of echinoderm species from the SFBR including published and unpublished records obtained through a comprehensive literature, museum collections, open-access databases records and recent samplings in the reserve. Future taxonomic and biogeographic studies will greatly benefit from this baseline of echinoderms species of these remote areas, which despite their strategic location have generally been included in inventories of countries or larger regions. The dataset presents the catalog numbers of the voucher for each species, facilitating any taxonomic revision that could be needed.

\section{DATA AVAILABILITY}

The dataset "Echinoderms from the SeaFlower Biosphere Reserve" that includes the complete list of species was assembled using the Darwin Core standard (DwC) and is available through the Integrated Publishing Tool of the OBIS and GBIF Colombian nodes (SIBM-SIB Colombia) (IPT SIBM OBIS Colombia link: https://ipt.biodiversidad.co/sibm/resource?r=echinoderms_from _the_seaflower_biosphere_reserve; DOI: https://doi.org/10. 15472/iipeom; GBIF: https://www.gbif.org/dataset/5a39c87c9d97-4282-b3a9-e2c292b4729d). Future updates of the list will be published in the latter repository. Changes incorporated to each new version of the list will be summarized in the respective metadata section of the electronic resource.

\section{AUTHOR CONTRIBUTIONS}

GHB-P, MB-S, NC, EG-G, BG, JM, and AA-H collected and identified echinoderm samples during the last expeditions to Quitasueño, Roncador, Serrana and Serranilla (2011, 2016 and 2017). GHB-P and MB-S collected and processed the information for the biological records and the checklist dataset. GHB-P compile the complete checklist dataset, performed the descriptive analyses and wrote the manuscript. GHB-P, MBS, NC, EG-G, BG, JM, and AA-H reviewed and approved the manuscript.

\section{FUNDING}

This work was supported by INVEMAR, Universidad Nacional de Colombia (UNAL), Comisión Colombiana del Océano, through Colombia BIO-Colciencias project, agreement No. 341 of 2017; and UNAL-Sede Caribe, Gobernación Departamental de San Andrés, Providencia y Santa Catalina and Corporación Autónoma Regional-CORALINA, agreement No. 021 of 2011. Projects involved: Aproximación a la diversidad de equinodermos en los cayos del norte, Reserva de Biosfera Seaflower-Universidad Nacional 2011; Estado del conocimiento de equinodermos en isla Cayo Serrana: biodiversidad y conectividad en el mar Caribe-Expedición Seaflower, 2016, Universidad Nacional de Colombia, INVEMAR; Caracterización de la epifauna y macrobentos $(0-800 \mathrm{~m})$ de Isla Cayo Serranilla y áreas aledañas-Expedición Seaflower 2017, Universidad Nacional de Colombia, INVEMAR; Evaluación física y biológica de las unidades ecológicas someras en la Isla Cayo Serranilla de la Reserva de Biósfera Seaflower-Expedicion SeaFlower, 2017, INVEMAR, Coralina. 


\section{ACKNOWLEDGMENTS}

We thank the people who organized and participated in SeaFlower Expeditions that helped enrich the inventories. T. Forbes, J. D. González, and V. Pizarro contributed with some photographic records. A. Polanco and A. Merchán

\section{REFERENCES}

Abril-Howard, A., Bolaños, N., Machacón, I., Lasso, J., Gómez, D. I., and Ward, V. (2012). Actualización del conocimiento de los ecosistemas marinos en la Reserva de Biósfera Seaflower, con énfasis en las islas de San Andrés y Providencia," in Atlas de la Reserva de Biósfera Seaflower. Archipiélago de San Andrés, Providencia y Santa Catalina, eds D. I. Gómez-López, C. Segura-Quintero, P. C. Sierra-Correa, and J. GarayTinoco (CORALINA-INVEMAR, 2012. Instituto de Investigaciones Marinas y Costeras "José Benito Vives De Andréis" -INVEMAR- y Corporación para el Desarrollo Sostenible del Archipiélago de San Andrés, Providencia y Santa Catalina-CORALINA, Serie de Publicaciones Especiales de INVEMAR 28), 129-157.

Alvarado-Barrientos, J. J., and Solís-Marín, F. A. (eds) (2013). Echinoderm Research and Diversity in Latin America. Berlin; Heidelberg: Springer-Verlag.

Benavides-Serrato , M., Borrero-Pérez, G. H., Merchán-Cepeda, A., and Campos, N. H. (2018b). Caracterización de la Epifauna y Macrobentos $(0-800 \mathrm{~m}) \mathrm{de}$ Isla Cayo Serranilla y Áreas aledañas: Equinodermos Primera Fase 2017. v1. Dataset/Occurrence. Universidad Nacional de Colombia. Available online at: https://ipt.biodiversidad.co/sibm/resource? $r=$ serranilla2017\&amp;v\$=\$1.0. doi: 10.15472/lbjyx0

Benavides-Serrato, M., Borrero-Pérez, G. H., Cantera, J. R., CohenRengifo, M., and Neira, R. (2013). "Echinoderms of Colombia," in Echinoderm Research and Diversity in Latin America, eds J. J. AlvaradoBarrientos, and F. A. Solís-Marín (Berlin; Heidelberg: Springer-Verlag), $145-182$.

Benavides-Serrato, M., Borrero-Pérez, G. H., and Díaz-Sánchez, C. M. (2011). Equinodermos del Caribe Colombiano I: Crinoidea, Asteroidea y Ophinoridea. Serie de Publicaciones Especiales de Invemar 22. Santa Marta.

Benavides-Serrato, M., Gavio, B., Galeano, E., Medina, J., and Abril-Horward, A. (2018a). Diversidad de Equinodermos en los Cayos del Norte, Reserva de Biósfera SeaFlower, 2011. v1. Dataset/Occurrence. Universidad Nacional de Colombia. Available online at: https://ipt.biodiversidad.co/sibm/resource? $\mathrm{r}=$ cayosnorte2011\&amp; $\$=\$ 1.0$. doi: $10.15472 /$ ygq6i9

Borrero-Pérez, G. H. (2018). Equinodermos_unidades_ecologicas_someras_ Serranilla2017-Seflower-Proyecto_Colombia_BIO. v1. Dataset/Occurrence. Instituto de Investigaciones Marinas y Costeras - INVEMAR. Available online at: https://ipt.biodiversidad.co/sibm/resource?r=equinodermos unidades_ecologicas_someras_serranilla2017-seaflower-proyecto_colombia_ bio. doi: 10.15472/kfevgp

Borrero-Pérez, G. H., Benavides-Serrato, M., and Díaz-Sánchez, C. M. (2012). Equinodermos del Caribe Colombiano II: Echinoidea y Holothuroidea. Serie de Publicaciones Especiales de Invemar No. 30. Santa Marta. Available online at: http://www.invemar.org.co/redcostera1/invemar/docs/10454EQII__ web.pdf

Borrero-Pérez, G. H., Díaz-Sánchez, C. M., and Benavides-Serrato, M. (2016). "Equinodermos. Invertebrados innovadores," in Biodiversidad del mar de Los Siete Colores, Serie de Publicaciones Generales del INVEMAR No. 84, Santa Marta - Colombia), eds M. D. Vides, E. C. Alonso, and N. Bolaños (Instituto de Investigaciones Marinas y Costeras-INVEMAR y Corporación para el Desarrollo Sostenible del Archipiélago de San Andrés, Providencia y Santa Catalina-CORALINA), 156-177. Available online at http://cinto.invemar.org. $\mathrm{co} /$ sai/app/pdf/biodiversidad-del-mar-de-los-siete-colores-web.pdf

Clark, A. M., and Downey, M. E. (1992). Starfishes of the Atlantic. Natural History Museum Publications. London: Chapman and Hall. helped during field trips and projects development. E. Montoya assisted the datasets elaboration (SIBM) and S. Millán, LABSIS-INVEMAR, helped with the map. Two reviewers for their suggestions to improve the manuscript. Contribution No. INVEMAR: 1215; and CECIMAR: 486.
Clark, H. (1939). Echinoderms (other than holothurians) collected on the Presidential cruise of 1938. Smithsonian Miscellaneous Collections, 98, 1-18.

CORALINA (2001). Plan de Manejo Ambiental Old Point Regional Mangrove Park 2001-2011. Documento Discusión. San Andrés: CORALINA.

CORALINA-INVEMAR (2012). "Atlas de la reserva de Biósfera Seaflower. Archipiélago de San Andrés, providencia y Santa Catalina," in Serie de Publicaciones Especiales de INVEMAR No. 28, eds D. I. GómezLópez, C. Segura-Quintero, P. C. Sierra-Correa, and J. Garay-Tinoco (Santa Marta: Instituto de Investigaciones Marinas y Costeras "José Benito Vives De Andréis"-INVEMAR y Corporación para el Desarrollo Sostenible del Archipiélago de San Andrés, Providencia y Santa CatalinaCORALINA) Available online at http://www.invemar.org.co/redcosteral/ invemar/docs/10447AtlasSAISeaflower.pdf

Diaz, J. M., Barrios, L. M., and Gomez, D. I. (eds). (2003). Las Praderas de Pastos Marinos de Colombia: Estructura y Distribución de un Ecosistema Estratégico. Serie Publicaciones Especiales No. 10. Santa Marta: INVEMAR.

García, M. I., McCormick, C., Chow, R., Peñaloza, G., Connolly, E., Mitchell, A., et al. (2003). Plan de Manejo del Área Marina Protegida Seaflower - Parte I. Proyecto Caribbean Archipelago Biosphere Reserve: Regional Marine Protected Area System CO-GM-P066646. San Andrés Isla San Andrés Isla: GEF-TOCCORALINA.

García-Hanse, I., and Álvarez-León, R. (2007). Macroflora y macrofauna asociadas al cordón arrecifal de Little Reef (Isla de San Andrés, Colombia). Revista Luna Azul 25, 61-77. doi: 10.17151/ luaz.2007.25.5

Guzman, H. M., and Guevara, C. (2002). Population structure, distribution and abundance of three commercial species of sea cucumber (Echinodermata) in Panama. Caribbean J. Sci. 38, 230-238.

Hendler, G., Miller, J., Pawson, D., and Porter, M. (1995). Echinoderms of Florida and the Caribbean Sea Stars, Sea Urchins and Allies. Washington, DC: Smithsonian Institution Press.

INVEMAR-ANH (2012). Línea Base Ambiental en el Área de Régimen Común Jamaica-Colombia Como Aporte al Aprovechamiento Sostenible de los Recursos Marinos Compartidos, Informe Técnico Final. Santa Marta: INVEMAR-ANH.

Lasso, Z. J. (2007a). Informe de gestión y acciones del Old Point Regional Mangrove Park. Proyecto "Protección y Conservación de Los Recursos de la Biodiversidad y de los Ecosistemas Estratégicos en la Reserva de la Biosfera Seaflower." Documento de Discusión. CORALINA.

Lasso, Z. J. (2007b). Las Acciones Desarrolladas por la Corporación Para Lograr la Recuperación, Conservación, Protección y uso Sostenible de los Recursos Naturales Presentes en los Manglares del Parque Regional Old Point, Incluyendo Evaluación Técnica y Participativa de la Implementación del Parque Regional. Proyecto Protección y Conservación de los recursos de la biodiversidad y de los ecosistemas estratégicos dentro de la Reserva de Biosfera Seaflower. Informe Técnico. CORALINA.

Lessios, H. A. (2016). The great Diadema antillarum die-off: 30 years later. Annu. Rev. Mar. Sci. 8, 1.1-1.17. doi: 10.1146/annurev-marine122414-033857

Mejía-Ladino, L. M., Gómez, D. I., Montoya-Cadavid, E., and NavasCamacho, R. (2008). Actualización de la Línea Base de Flora y Fauna Marina y Costera del Parque Regional Old Point. Informe Técnico Final. Santa Marta. 
Orrell, T., and Hollowell, T. (2018). NMNH Extant Specimen Records. Version 1.19. Occurrence dataset. National Museum of Natural History, Smithsonian Institution. Available online at: https://doi.org/10.15468/hnhrg3 accessed via GBIF.org on 2018-07-23.

Prestridge, H. (2016). Biodiversity Research and Teaching Collections - TCWC Marine Invertebrates. Version 5.1. Occurrence Dataset. Texas A\&M University Biodiversity Research and Teaching Collections. Available online at https://doi.org/10.15468/dfrwoh accessed via GBIF.org on 2018-07-23.

Quiñones, R. (1981). "Lista preliminar de los equinodermos," in Informe Sobre los Resultados de la Expedición a las Islas de Providencia y Santa Catalina, ed B. Werding, J. Garzón, and S. Zea (Santa Marta: Invemar), $35-45$.

Toral-Granda, V. (2008). "Population status, fisheries and trade of sea cucumbers in Latin America and the Caribbean," in A Global Review of Fisheries and Trade Sea Cucumbers, eds V. Toral-Granda, A. Lovatelli, and M. Vasconcellos (Rome: AO Fisheries and Aquaculture Technical Paper. No. 516), 211-229.

UNAL (Universidad Nacional de Colombia), Instituto de Investigaciones Marinas y Costeras "José Benito Vives de Andreis", Universidad del Magdalena, Universidad Jorge Tadeo Lozano, Pontifica Universidad Javeriana, Fundación Malpelo y Otros Ecosistemas Marinos, et al. (2016). Caracterización de equinodermos de Serrana durante la Expedición Seaflower 2016. Versión 1.1. 39 registros, aportados por: Benavides M, Campos N, Bustos D, Perez D,
Howard A, Pizarro D, Forbes, T, González JD. Conjunto de datos/Registros biológicos. Available online at: https://ipt.biodiversidad.co/sib/resource? $\mathrm{r}=$ unal_equinodermos_seaflower_2016. doi: 10.15472/3fz5b3

Vega-Sequeda, J., Díaz-Sánchez, C. M., Gómez-Campo, K., López-Londoño, T., Díaz-Ruiz, M., and Gómez-López, D. I. (2015). Biodiversidad marina en Bajo Nuevo, Bajo Alicia y Banco Serranilla, Reserva de Biosfera Seaflower. Boletín Investigaciones Marinas Costeras 44, 199-224. Available online at: http://cinto.invemar.org.co/ojs/index.php/boletin/article/view/27/ 26. doi: $10.25268 /$ bimc.invemar.2015.44.1.27

Vélez, C. (2003). Estrellas Quebradizas Infaunales (Echinodermata: Ophiuroidea) Asociadas a Fondos Blandos Someros de las Islas de San Andrés y Providencia. Bogotá: Tesis Biólogo Marino, Universidad de Bogotá Jorge Tadeo Lozano.

Conflict of Interest Statement: The authors declare that the research was conducted in the absence of any commercial or financial relationships that could be construed as a potential conflict of interest.

Copyright (๑ 2019 Borrero-Pérez, Benavides-Serrato, Campos, Galeano-Galeano, Gavio, Medina and Abril-Howard. This is an open-access article distributed under the terms of the Creative Commons Attribution License (CC BY). The use, distribution or reproduction in other forums is permitted, provided the original author(s) and the copyright owner(s) are credited and that the original publication in this journal is cited, in accordance with accepted academic practice. No use, distribution or reproduction is permitted which does not comply with these terms. 